\title{
Advances in surgery for pancreatic cancer
}

\author{
Alexandra W. Acher ${ }^{1}$, Josh Bleicher ${ }^{1}$, Austin Cannon ${ }^{1}$, Courtney Scaife ${ }^{2}$ \\ ${ }^{1}$ Department of General Surgery, University of Utah Health Care, Salt Lake City, UT, USA; ${ }^{2}$ Huntsman Cancer Institute, University of Utah, Salt \\ Lake City, UT, USA \\ Contributions: (I) Conception and design: All authors; (II) Administrative support: C Scaife; (III) Provision of study materials or patients: None; (IV) \\ Collection and assembly of data: AW Acher, J Bleicher, A Cannon; (V) Data analysis and interpretation: None; (VI) Manuscript writing: All authors; \\ (VII) Final approval of manuscript: All authors. \\ Correspondence to: Josh Bleicher. Department of General Surgery, University of Utah Health Care, 50 N Medical Drive, Salt Lake City, UT 84112 , \\ USA. Email: Josh.bleicher@hsc.utah.edu.
}

\begin{abstract}
Over the past 135 years, the field of pancreatic surgery for treatment of pancreatic malignancies has been a challenge to the surgical community. Originally filled with unacceptably high morbidity and mortality, these obstacles have been overcome through the work of numerous great surgeons in recent decades. Today, despite the improved safety of operating on the pancreas, patients still suffer from high rates of malignant recurrence and poor overall survival. Recent advances in pancreatic surgery aim to further improve the morbidity of these operations while increasing the number of patients who are both candidates for surgical resection and those who receive complete resections. This review focuses on recent literature describing the pros and cons of minimally invasive approaches to pancreatic surgery and the risks and benefits of vascular reconstruction to improve resectability. Both topics are currently debated amongst pancreatic surgeons and this article summarizes the varied viewpoints and their impact on outcomes in pancreas cancer surgery.
\end{abstract}

Keywords: Pancreas surgery; Whipple; minimally invasive surgery; vascular reconstruction

Submitted Mar 16, 2018. Accepted for publication Apr 20, 2018.

doi: 10.21037/jgo.2018.05.05

View this article at: http://dx.doi.org/10.21037/jgo.2018.05.05

\section{Introduction}

The first surgical procedure for resection of pancreatic cancer, a distal pancreatectomy and splenectomy, was performed by Freidrich Trendelenberg in 1882 (1). Unfortunately, this patient died from postoperative complications. Alessandro Codivilla performed the first pancreaticoduodenectomy 6 years later; however, this patient also died from surgical complications (2). Pancreaticoduodenectomy was performed by few other surgeons prior to being defined, then refined, by Whipple et al. in 1935 (3). Despite advances in surgery for pancreatic carcinoma, the mortality of patients undergoing pancreaticoduodenectomy persisted around $30 \%$ into the 1960 s, with postoperative morbidity as high as $60 \%$ (4). This high complication rate and an overall 5 -year survival rate of $0.4 \%$ during the 50 years following Whipple's first operation led some to conclude "that the notion of 'resections for cure'... should be abandoned" $(5,6)$. Over the next 30 years, technical success has improved significantly. In 2006, Cameron et al. revealed a 1\% 30-day or in-hospital mortality rate on his personal series of 1,000 pancreaticoduodenectomies, setting a new standard for pancreatic surgeons (7). Despite improved technical success however, 5-year overall survival for pancreatic cancer patients remains low $(8,9)$.

Recent trends in pancreas cancer surgery include the increased use of minimally invasive techniques. The first laparoscopic pancreatic surgery was a pylorussparing Whipple in 1992 with multiple laparoscopic distal pancreatectomies performed in subsequent years (10). All of these early procedures were performed for benign indications rather than oncologic resection. More recently, robotic pancreatectomies have been performed with some advocating 
equivalent outcomes and decreased rates of conversion to open procedures compared to laparoscopic surgeries (11). Evidence on the advantages of laparoscopic and robotic pancreatic surgery remains mixed, and widespread adoption of a minimally invasive approach has yet to take hold, particularly for oncologic operations $(12,13)$.

Advances in vascular resection and reconstruction have also allowed for evolution in pancreas cancer surgery (14). The definition of borderline resectable and unresectable disease are evolving with advances in preoperative imaging, neoadjuvant therapies, and vascular resection and reconstruction options. More research regarding the safety and efficacy of vascular resection and its impact on outcome is needed before definitive conclusions can be generated regarding the utility of vascular resection $(15,16)$.

This review summarizes the recent advances in surgery for pancreatic cancer, focusing on the role of minimally invasive techniques to improve surgical outcomes and vascular resection and reconstruction to increase rates of tumor resectability.

\section{Minimally invasive surgery for pancreatic cancer}

Over the last several decades, minimally invasive approaches to pancreatic surgery have become more common. The popularization of minimally invasive techniques was founded in the desire for improved cosmetic incisions and the assumption of reduced hospital stays, shorter overall recovery times, and decreased post-operative pain. Innovation in laparoscopic, and more recently, robotic surgery has led to increasing numbers of minimally invasive pancreatic surgeries; however, more research is required regarding the recovery outcomes, safety, oncologic outcomes, costs, and necessary training and certification before minimally invasive techniques can be recommended for widespread adoption.

\section{Distal pancreatectomy}

The first laparoscopic distal pancreatectomy was described in 1992 (10). With no anastomosis and a relatively less challenging dissection compared to other pancreatic resections, the laparoscopic distal pancreatectomy is now commonly used for both benign and malignant pancreatic tail lesions. No randomized trials comparing laparoscopic versus open distal pancreatectomy exist; however, retrospective case series have implied reduced blood loss, shorter hospital stays, and lower rates of surgical site infections with a laparoscopic approach for both benign and malignant disease $(17,18)$. The rate of significant morbidity, including pancreatic fistula, is equivalent in these two techniques (19). For benign pathologies, these outcomes make laparoscopy an attractive option. The same observations are true for resectable malignant lesions; however, oncologic outcomes have not been studied in a prospective manner. For malignant pancreatic lesions, small retrospective studies demonstrate similar benefits (reduced blood loss, shorter length of stay, lower rate of surgical site infection) as laparoscopy for benign lesions, and show similar rates of R0 resections (20). Prospective, randomized data is needed to better compare open $v$ s. laparoscopic outcomes in surgeries for malignant disease.

More recently, robotic approaches have been used to perform distal pancreatectomies for treatment of both benign and malignant diseases of the pancreas. Evidence on differences between the laparoscopic and robotic approach to distal pancreatectomy is minimal and limited to a few retrospective studies. Proponents of the robotic approach argue it can provide improved visualization and decreased intraoperative blood loss when compared to the laparoscopic approach (21). There is retrospective evidence showing no significant differences in achievement of R0 resection, development of pancreatic fistula, or mortality between laparoscopic and robotic approaches $(22,23)$. Available evidence also demonstrates equivalent perioperative morbidity and mortality for the robotic approach compared to an open approach (24). This data is severely limited by the low volume, retrospective, single center (often single surgeon) nature of available studies leading to the potential for a significant selection bias $(25,26)$.

\section{Pancreaticoduodenectomy}

Minimally invasive techniques for the pancreaticoduodenectomy have also been explored in recent years. The first laparoscopic pancreaticoduodenectomy lasted over 20 hours, with the patient requiring a 30-day post-operative hospital stay (27). Since the introduction of the laparoscopic pancreaticoduodenectomy, a single-institution retrospective study has shown decreased transfusion requirements, shorter hospital times, and shorter time to adjuvant chemotherapy compared to the open approach (22). All laparoscopic cases in this series were performed by a single expert surgeon at a high-volume institution, limiting the generalizability. Other studies have similar limitations. The robotic platform has also been employed for the pancreaticoduodenectomy, with different studies arriving 
at different conclusions. One study shows significantly longer length of stay, higher incidence of post-operative pancreatic fistula, and increased rate of conversion to an open operation in the robotic group compared to laparoscopy (28). Another larger study demonstrates comparable outcomes of robotic versus laparoscopic pancreaticoduodenectomy and decreased rates of conversion to open surgery, incidence of pancreatic fistula, and total blood loss with increasing experience with the robotic platform (23). Evidence implies equivalent short-term post-operative outcomes for the laparoscopic compared to open approach, while data is varied regarding the robotic approach. Again, available data is severely limited by the small sample sizes and significant selection bias, limiting the ability to draw meaningful conclusions from currently available evidence (25). More research is required to determine the difference in oncologic outcomes between these approaches.

\section{Costs and training}

The data on costs of minimally invasive pancreatic surgery is mixed and depends on the methodology used to determine costs $(24,29)$. According to some studies, if cost savings related to reduced hospital days and improved recovery time are included, the laparoscopic and robotic technique may be comparable to the traditional open approach (30). Other studies demonstrate increased costs for minimally invasive approaches (31). Studies uniformly demonstrate significantly increased operative costs with minimally invasive approaches, some of which can be balanced out by reduced post-operative costs. Many of these studies contain significant selection bias by comparing a minimally invasive patient cohort with benefit from modern bundles for early recovery after surgery to historical groups of patients in the open surgery cohort that did not benefit from these enhanced recovery techniques (32). More research is required to accurately identify the costs of minimally invasive pancreatic surgery using an appropriate control group.

Another consideration in the increasing adoption of laparoscopic and robotic approaches to pancreas surgery is surgeon and institutional experience and certification. In general, minimally invasive pancreaticoduodenectomies remain limited to high volume pancreas cancer centers. This is in part due to the steep learning curve associated with minimally invasive pancreatic surgery. A surgeon needs to perform approximately 70 robotic pancreatic surgeries to be considered proficient (33). Achieving this volume is difficult; the average hepatopancreatobiliary or surgical oncology fellow performs 60 or fewer total pancreatic operations during their advanced training (34). Achieving safe training in minimally invasive techniques for widespread adaptation presents a significant challenge if this practice is to gain favor.

\section{Summary}

A growing body of evidence exists demonstrating the equivalence or benefit of minimally invasive pancreatic surgery compared to the open approach regarding safety and short-term peri-operative outcomes. Data on oncologic outcomes between open and minimally invasive approaches is sparser. Additionally, more research on the costs and necessary training for minimally invasive pancreatic surgery is required to ensure the costs of widespread adoption of this approach to pancreatic surgery is justified. While there is a fair amount of data available, the quality of this data is heavily biased and methodology often lacking based on the limitations described above. An expert panel on this topic recommends only "the continued development of minimally invasive pancreatic resection by experienced surgeons in high-volume pancreatic centres" (32). Several randomized controlled trials are ongoing and the results of these studies may help clarify the costs and benefits of minimally invasive approaches (25).

\section{Redefining resectability with vascular reconstruction}

An additional advance in pancreatic surgery has involved vascular resection and reconstruction to increase the number of patients with resectable disease. The National Comprehensive Cancer Network guidelines define borderline resectable disease as solid tumor contact with the superior mesenteric vein (SMV) or portal vein (PV) of greater than 180 degrees, solid tumor contact with the inferior vena cava (IVC), tumor contact with the common hepatic artery without extension to the celiac axis or hepatic artery bifurcation, or contact with the superior mesenteric artery (SMA) of greater than 180 degrees (35). Greater than 180 degrees of SMA or celiac axis contact, or unreconstructible SMV or PV due to tumor involvement or thrombus is classified as unresectable disease. With advances in preoperative imaging modalities, evolving and more effective neoadjuvant therapies, specialization of cancer surgery to large volume cancer centers, and advances in surgical options for safe vascular resection and reconstruction, tumors previously considered borderline or unresectable are now 
potentially resectable in specialized centers (36).

Preoperative computed tomography (CT) imaging is often inaccurate in assessment of vascular involvement (37). The discrepancy in preoperative imaging and intraoperative assessment of resectability necessitates intraoperative decisions regarding vascular resection and reconstruction. In pancreas cancer surgery, there is limited data regarding the incidence of arterial resection; however, the incidence of venous resection ranges from $40-70 \%$ in high volume cancer centers in the US (36). Despite these high resection rates, available data demonstrates that only $20-70 \%$ of resected specimens reveal histological evidence of vascular invasion $(38,39)$. Although some of this discrepancy may be due to the influence of neoadjuvant therapies and regression of tumor from vascular structures, it also suggests that preoperative imaging as well as intraoperative evaluation is not effective at distinguishing malignant invasion from the desmoplastic reaction that accompanies diseaseassociated inflammation and neoadjuvant treatment. With advancements in surgical options, it is important to recognize borderline resectable disease preoperatively to enhance the use of neoadjuvant therapy and ultimately the potential for resectability. Although the majority of data on perioperative morbidity and mortality, as well as overall mortality, considers arterial and venous resection together, it may be important to consider these separately as they have different implications for operative planning and perioperative morbidity and mortality (36,40-42).

\section{Arterial reconstruction}

Arterial involvement of the SMA, celiac axis, and hepatic artery has generally been considered unresectable disease due to life-threatening perioperative complications such as bowel ischemia, reoperation, hemorrhage, and SMA thrombosis associated with resection. These complications range from $39-91 \%$ in systematic reviews $(41,42)$. Older, heterogeneous, retrospective data suggests that arterial resection has worse mortality and morbidity than venous resection. This comparison, however, is not generated from data comparing equivalent populations or disease burdens. This older data, as well as newer data, demonstrates that when outcomes are compared between arterial resection $v s$. palliative surgery for disease that would have otherwise been considered unresectable, there is improved survival in the group undergoing arterial resection (42). Interestingly, retrospective data from more recent single institution studies suggests that despite increased morbidity, there is no difference in mortality associated with en bloc venous and arterial resection and reconstruction compared with operations done for resectable disease $(40,43)$. Additionally, progress in neoadjuvant therapies has allowed for downstaging of tumors with arterial invasion to borderline resectable or resectable disease making surgical resection more achievable (44). Despite these advancements, it is currently accepted that arterial reconstruction is only appropriate in highly selected patients in high volume specialty cancer centers with surgeons who are familiar with the advanced techniques required for reconstruction.

\section{Venous reconstruction}

Similarly, the data available on venous resection and reconstruction is limited to evidence from largely retrospective and heterogeneous studies $(39,40,43,44)$. Within this limited data, venous resection and reconstruction to achieve complete resection of pancreas cancer has been found to be safe, with no difference in overall survival and morbidity when compared to surgeries for resectable disease that did not require venous resection $(36,39,40,43)$. Similar to arterial resection, venous resection and reconstruction has also been shown to improve survival when compared with palliative surgeries for what would otherwise be considered unresectable disease $(36,39,40,43,44)$.

\section{Summary}

Although the incidence of vascular resection and reconstruction in pancreas cancer surgery is increasing, data regarding outcomes is largely retrospective, heterogeneous, and inadequate to completely inform data driven conclusions. Additionally, despite the different technical implications of venous and arterial resection, distinction between these resections and their outcomes is lacking in most current data on vascular resection. Retrospective studies demonstrate no difference in morbidity and mortality for venous reconstruction when compared with surgeries for tumor resections without vascular involvement $(36,39,40,43)$. The available data on arterial resection is less clear, with some older studies citing worse morbidity and mortality while more recent studies site at least equivalent mortality despite a persistent morbidity burden $(41,42)$. More research is required on outcomes regarding perioperative morbidity and mortality and overall survival following vascular reconstruction before a recommendation on routine use can be made; there may be benefit to 
examining arterial and venous reconstruction separately. No ongoing trials currently exist evaluating this question. More research on methods to enhance pre- and intra-operative recognition of vascular invasion is also needed, particularly given the difficulty in distinguishing between tumor and peri-tumoral desmoplastic reaction and inflammation on imaging. In specialized centers with surgeons who are trained in advanced vascular resection and reconstruction techniques, vascular resection and reconstruction should be considered individually for patients as it may offer equivalent morbidity and mortality as resection without vascular involvement and a potential survival benefit over what would otherwise be either a palliative operation or nonoperative management of unresectable disease.

\section{Conclusions}

Pancreatic surgery has made major advances in the past 135 years. Recent trends have focused on decreasing the morbidity of pancreatic surgery through minimally invasive techniques and redefining resectability through advanced vascular reconstruction and use of neoadjuvant therapies. While minimally invasive techniques for pancreatic tail surgeries are now largely accepted, laparoscopic and robotic techniques for disease involving the pancreatic head remains an evolving field limited to high volume, specialized cancer centers. Significantly more evidence is needed to determine the risks and benefits of these approaches, particularly for the more expensive robotic approach, before this can be recommended for routine care.

Surgical management of pancreatic cancer remains the only chance for cure and achieving an $\mathrm{R} 0$ margin is associated with improved overall and disease-free survival (45). Vascular reconstruction is a promising tool to increase complete tumor resection. Better data from well-designed studies regarding the incidence of vascular invasion and the perioperative and oncologic impacts of vascular resection and reconstruction are needed to definitively comment on the utility of vascular resection and reconstruction.

\section{Acknowledgements}

None.

\section{Footnote}

Conflicts of Interest: The authors have no conflicts of interest to declare.

\section{References}

1. Griffin JF, Poruk KE, Wolfgang CL. Pancreatic cancer surgery: past, present, and future. Chin J Cancer Res 2015;27:332-48.

2. Schnelldorfer T, Sarr M. Alessandro Codivilla and the First Pancreatoduodenectomy. Arch Surg 2009;144:1179-84.

3. Whipple AO, Parsons WB, Mullins CR. Treatment of carcinoma of the ampulla of Vater. Ann Surg 1935;102:763-79.

4. Lillemoe KD. Current Management of Pancreatic Carcinoma. Ann Surg 1995;221:133-48.

5. Gudjonsson B. Cancer of the Pancreas: 50 Years of Surgery. Cancer 1987;60:2284-303.

6. Crile G Jr. The advantages of bypass operations over Whipple. Surg Gynecol Obstet 1970;130:1049-53.

7. Cameron JL, Riall TS, Coleman J, et al. One Thousand Consecutive Pancreaticoduodenectomies. Ann Surg 2006;244:10-5.

8. Siegel RL, Miller KD, Jemal A. Cancer Statistics, 2018. CA Cancer J Clin 2018;68:7-30.

9. Ryan DP, Hong TS, Bardeesy N. Pancreatic Adenocarcinoma. N Engl J Med 2014;371:1039-49.

10. Cuschieri A, Jakimowicz JJ, van Spreeuwel J. Laparoscopic Distal 70\% Pancreatectomy and Splenectomy for Chronic Pancreatitis. Ann Surg 1996;223:280-5.

11. Liu R, Liu Q, Zhao Z, et al. Robotic versus laparoscopic distal pancreatectomy: A propensity score-matched study. J Surg Oncol 2017;116:461-9.

12. Cesaretti M, Bifulco L, Costi R, et al. Pancreatic resection in the era of laparoscopy : State of Art. A systematic review. Int J Surg 2017;44:309-16.

13. Xourafas D, Ashley SW, Clancy TE. Comparison of Perioperative Outcomes between Open, Laparoscopic, and Robotic Distal Pancreatectomy: a Analysis of 1815 Patients from the ACS-NSQIP ProcedureTargeted Pancreatectomy Database. J Gastrointest Surg 2017;21:1442-52.

14. Maley WR, Yeo CJ. Vascular Resections During the Whipple Procedure. Adv Surg 2017;51:41-63.

15. Sgroi MD, Narayan RR, Lane JS, et al. Vascular reconstruction plays an important role in the treatment of pancreatic adenocarcinoma. J Vasc Surg 2015;61:475-80.

16. Kasumova GG, Conway WC, Tseng JF. The Role of Venous and Arterial Resection in Pancreatic Cancer Surgery. Ann Surg Oncol 2018;25:51-8.

17. de Rooij T, Jilesen AP, Boerma D, et al. A Nationwide Comparison of Laparoscopic and Open Distal 
Pancreatectomy for Benign and Malignant Disease. J Am Coll Surg 2015;220:263-70.e1.

18. Nakamura M, Wakabayashi G, Miyasaka Y, et al. Multicenter comparative study of laparoscopic and open distal pancreatectomy using propensity score-matching. J Hepatobiliary Pancreat Sci 2015;22:731-6.

19. Mehrabi A, Hafezi M, Arvin J, et al. A systematic review and meta-analysis of laparoscopic versus open distal pancreatectomy for benign and malignant lesions of the pancreas: It's time to randomize. Surgery 2015;157:45-55.

20. Magge D, Gooding W, Coudry H, et al. Comparative Effectiveness of Minimally Invasive and Open Distal Pancreatectomy for Ductal Adenocarcinoma. JAMA Surg 2013;148:525-31.

21. Lai EC, Tang CN. Robotic distal pancreatectomy versus conventional laparoscopic distal pancreatectomy: a comparative study for short-term outcomes. Front Med 2015;9:356-60.

22. Croome KP, Farnell MB, Que FG, et al. Total Laparoscopic Pancreaticoduodenectomy for Pancreatic. Ann Surg 2014;260:633-8; discussion 638-40.

23. Boone BA, Zenati M, Hogg ME, et al. Identification of the Learning Curve. JAMA Surg 2015;150:416-22.

24. Lee SY, Allen PJ, Sadot E, et al. Distal Pancreatectomy: A Single Institution's Experience in Open, Laparoscopic, and Robotic Approaches. J Am Coll Surg 2015;220:18-27.

25. Barkun J, Fisher W, Davidson G, et al. Research considerations in the evaluation of minimally invasive pancreatic resection (MIPR). HPB (Oxford) 2017;19:246-53.

26. Vollmer CM, Asbun HJ, Barkun J, et al. Proceedings of the first international state-of-the-art conference on minimally-invasive pancreatic resection (MIPR). HPB (Oxford) 2017;19:171-7.

27. Gagner M, Pomp A. Laparoscopic pylorus-preserving pancreatoduodenectomy. Surg Endosc 1994;8:408-10.

28. Orti-Rodriguez R, Rahman S. A Comparative Review Between Laparoscopic and Robotic Pancreaticoduodenectomies. Surg Laparosc Endosc Percutan Tech 2014;24:103-8.

29. Ielpo B, Duran H, Diaz E, et al. Robotic versus laparoscopic distal pancreatectomy: A comparative study of clinical outcomes and costs analysis. Int J Surg 2017;48:300-4.

30. Baker EH, Seshadri R. Robotic pancreaticoduodenectomy : comparison of complications and cost to the open approach. Int J Med Robot 2016;12:554-60.

31. Tan CL, Zhang H, Peng B, et al. Outcome and costs of laparoscopic pancreaticoduodenectomy during the initial learning curve vs laparotomy. World J Gastroenterol 2015;21:5311-9.

32. Conlon KC, de Rooij T, van Hilst J, et al. Minimally invasive pancreatic resections: cost and value perspectives. HPB (Oxford) 2017;19:225-33.

33. Narula VK, Mikami DJ, Melvin WS. Robotic and Laparoscopic Pancreaticoduodenectomy A Hybrid Approach. Pancreas 2010;39:160-4.

34. Tseng JF, Pisters PWT, Lee JE, et al. The learning curve in pancreatic surgery. Surgery 2007;141:694-701.

35. National Comprehensive Cancer Network. Pancreatic Adenocarcinoma 2017. Available online: https://www.trikobe.org/nccn/guideline/pancreas/english/pancreatic.pdf

36. Helmink BA, Snyder RA, Idrees K, et al. Advances in the Surgical Management of Resectable and Borderline Resectable Pancreas Cancer. Surg Oncol Clin N Am 2016;25:287-310.

37. Allen VB, Gurusamy KS, Takwoingi Y, et al. Diagnostic accuracy of laparoscopy following computed tomography (CT) scanning for assessing the resectability with curative intent in pancreatic and periampullary cancer. Cochrane Database Syst Rev 2016;7:CD009323.

38. Tielsch JM, Katz J, Thulasiraj RD, et al. Exposure to indoor biomass fuel and tobacco smoke and risk of adverse reproductive outcomes, mortality, respiratory morbidity and growth among newborn infants in south India. Int J Epidemiol 2009 Oct;38:1351-63.

39. Yekebas EF, Bogoevski D, Cataldegirman G, et al. En bloc vascular resection for locally advanced pancreatic malignancies infiltrating major blood vessels: perioperative outcome and long-term survival in 136 patients. Ann Surg 2008;247:300-9.

40. Martin RC 2nd, Scoggins CR, Egnatashvili V, et al. Arterial and venous resection for pancreatic adenocarcinoma: operative and long-term outcomes. Arch Surg 2009;144:154-9.

41. Jegatheeswaran S, Baltatzis M, Jamdar S, et al. Superior mesenteric artery (SMA) resection during pancreatectomy for malignant disease of the pancreas: a systematic review. HPB (Oxford) 2017;19:483-90.

42. Mollberg N, Rahbari NN, Koch M, et al. Arterial resection during pancreatectomy for pancreatic cancer: a systematic review and meta-analysis. Ann Surg 2011;254:882-93.

43. Bockhorn M, Burdelski C, Bogoevski D, et al. Arterial en bloc resection for pancreatic carcinoma. Br J Surg 2011;98:86-92.

44. Kluger MD, Rashid F, Rosaria VL, et al. Resection of Locally Advanced Pancreatic Cancer without Regression 
of Arterial Encasement After Modern-Era Neoadjuvant

Therapy. J Gastrointest Surg 2018;22:235-41.

45. Ghaneh P, Kleeff J, Halloran CM, et al. The Impact of Positive Resection Margins on Survival and Recurrence

Cite this article as: Acher AW, Bleicher J, Cannon A, Scaife C. Advances in surgery for pancreatic cancer. J Gastrointest Oncol 2018;9(6):1037-1043. doi: 10.21037/jgo.2018.05.05
Following Resection and Adjuvant Chemotherapy for Pancreatic Ductal Adenocarcinoma. Ann Surg 2017. [Epub ahead of print]. 